\title{
Double-OAM-mode resistor loaded microstrip antenna with a top dielectric layer
}

\author{
Dang Weiguo, Zhu Yongzhonga), Yu Yang, and Zuo Kaiwei \\ Department of Information Engineering, Engineering University of PAP, \\ Wujing Road, Weiyang District, Xi'an City, Shaanxi Province 710086, China
}

a)bsbs1980@sina.com

\begin{abstract}
A double-Orbital-Angular-Momentum (OAM)-mode microstrip antenna is designed, simulated, fabricated and measured, which generates OAM with mode number $l=2$ and 3 on $5 \mathrm{GHz}$ by two resistor loaded microstrip rings respectively. For existing OAM antennas, structures like circular array, parabolic reflector and electromagnetic meta-surface are widely adopted, which are electrically large and hard to be integrated. However, the proposed antenna has a compact size and a low profile, which can be integrated in RF circuit as a component. To solve the problem of low gain in microstrip OAM antenna, parameters are optimised and a top dielectric layer is adopted, and its measured peak gain reaches $1.5 \mathrm{~dB}$ for $l=2$ and $1.8 \mathrm{~dB}$ for $l=3$. The measured frequency band, on which a near field vortex phase is observed and orbital angular momentum is generated, is 4.65-5.25 GHz for $l=2$, and $4.5-5.2 \mathrm{GHz}$ for $l=3$. Besides, this antenna can multiplex more OAM modes by adopting more microstrip rings.
\end{abstract}

Keywords: OAM, microstrip antenna, integration, gain, multi-mode Classification: Microwave and millimeter-wave devices, circuits, and modules

\section{References}

[1] B. Thidé, et al:: "Utilization of photon orbital angular momentum in the lowfrequency radio domain," Phys. Rev. Lett. 99 (2007) 087701 (DOI: 10.1103/ PhysRevLett.99.087701).

[2] F. Tamburini, et al:: "Encoding many channels on the same frequency through radio vorticity: First experimental test,” New J. Phys. 14 (2012) 033001 (DOI: 10.1088/1367-2630/14/3/033001).

[3] S. M. Mohammadi, et al.: "Orbital angular momentum in radio-A system study," IEEE Trans. Antennas Propag. 58 (2010) 565 (DOI: 10.1109/TAP.2009. 2037701).

[4] B. Liu, et al:: "A broadband dual-polarized dual-OAM-mode antenna array for OAM communication,” IEEE Antennas Wireless Propag. Lett. 16 (2017) 744 (DOI: 10.1109/LAWP.2016.2601615).

[5] W. Zhang, et al.: "Four-OAM-mode antenna with traveling-wave ring-slot structure," IEEE Antennas Wireless Propag. Lett. 16 (2017) 194 (DOI: 10. 1109/LAWP.2016.2569540).

[6] C. Deng, et al.: "Generation of OAM radio waves using circular Vivaldi antenna array," Int. J. Antennas Propag. 2013 (2013) 847859 (DOI: 10.1155/ 
2013/847859).

[7] Y. Zhang, et al.: "Design of planar circular loop microstrip integrated radio frequency OAM antenna" (2015) http://www.paper.edu.cn.

[8] Y. Chen, et al:: "Half-mode substrate integrated waveguide antenna for generating multiple orbital angular momentum modes," Electron. Lett. 52 (2016) 684 (DOI: 10.1049/el.2015.4416).

[9] L. Gui, et al.: "Circular slot antenna systems for OAM waves generation," IEEE Antennas Wireless Propag. Lett. 16 (2016) 1443 (DOI: 10.1109/LAWP. 2016.2641458).

[10] S. Zheng, et al:: "Transmission characteristics of a twisted radio wave based on circular traveling-wave antenna," IEEE Trans. Antennas Propag. 63 (2015) 1530 (DOI: 10.1109/TAP.2015.2393885).

[11] W. Yang: "Study on microstrip patch antenna with high permittivity superstrata," J. Microwares 16 (2000) 610.

\section{Introduction}

As an intrinsic property of electromagnetic waves, Orbital Angular Momentum (OAM) can theoretically multiplex infinite orthogonal channels on a single frequency, greatly relieving the scarcity of radio frequency spectrum resource, thus it has aroused widespread concern, and the design of practical OAM antenna is becoming current focus $[1,2]$. To implement the great potential of OAM and make it widely applied in various of miniaturized electric devices, a practical OAM antenna is required, which has small size and low profile, enough gain, and the ability to multiplex different OAM modes within a single band. However, due to the rotating nature of OAM, radiation patterns of all the OAM antennas are centrally-hollowed, leading to dispersed radiating power and low gain, compared with non-OAM antennas [3]. To deal with this problem, antenna arrays and reflectors are currently widely used in OAM antennas $[4,5,6]$, which lead to large size and high profile. Except for these two kinds, the gain of current OAM antennas, especially the substrate-integrated ones, are usually as low as about $0 \mathrm{~dB}$ $[7,8,9]$. Furthermore, the demand of multiplexing several OAM modes on the same frequency also makes miniaturization and integration harder. Due to the contradiction between gain and size, practical applications of current OAM antennas are limited.

Theoretical analysis shows that besides the propagating phase dependency $e^{-j \beta r}$, a circular loop with a traveling-wave current distribution can also generate a vortex phase dependency $e^{j \varphi l}$ in its radiating electromagnetic field formulations, as long as its circumference is $l$ multiple of the travelling wavelength. And under the joint effect of $e^{j \varphi l}$ and $e^{-j \beta r}$, a phase vortex with $l$ arms can be observed on a plane perpendicular to the propagating direction, which is the characteristic of OAM waves [10]. Based on this principle, a highly integrated microstrip ring OAM antenna has been proposed [7]. However, it is basically a circular transmission line and no radiation-improving measure is taken, and its peak gain is far below $0 \mathrm{~dB}$.

The double-OAM-mode antenna in this letter uses microstrip ring with a to generate OAM with mode number $l=2$ and 3 respectively, both on $5 \mathrm{GHz}$. 
Besides, the line width of microstrip and substrate thickness are optimised, and a top dielectric layer is adopted, resulting in higher radiation efficiency and gain. Measurement shows that its peak gain is $1.5 \mathrm{~dB}$ for $l=2$, and $1.8 \mathrm{~dB}$ for $l=3$, increased by more than $10 \mathrm{~dB}$ comparing to the simulating result of the initial model which takes no measures for radiation enhancement. The proposed antenna is compactly-structured and easy to be integrated in RF circuit, with enough gain for practical application. By adopting more microstrip rings, it can also multiplex more OAM modes.

\section{Antenna configurations and working principle}

This antenna consists of two layers, namely a circular microstrip layer and a top dielectric layer. Both layers are FR4 epoxy, with $\varepsilon_{r}=4.4$ and loss tangent $\tan \delta=0.02$. On the microstrip layer, two microstrip rings, whose characteristic impedance is $50 \Omega$, are both chipped and loaded by matched loads whose impedance is $R_{L}$ terminally. The rings are respectively fed by feeding pins that are connected to $50 \Omega$ coaxial line's inner conductors. Circumference of the inner ring is about $2 \lambda_{g}$, while the outer is about $3 \lambda_{g}$. With proper circumferences and matched terminal loads, circular travelling waves can be excited on the microstrips, and OAM with $l=2$ and 3 can be generated respectively by the two rings, according to the principle introduced in the forward section. Another layer is a top dielectric layer covering on antenna's upper surface, which can increase its radiation efficiency and gain. Besides, as shown in Fig. 1, simulating model in ANSYS HFSS 18.0 of this proposed antenna is placed on XY plane.

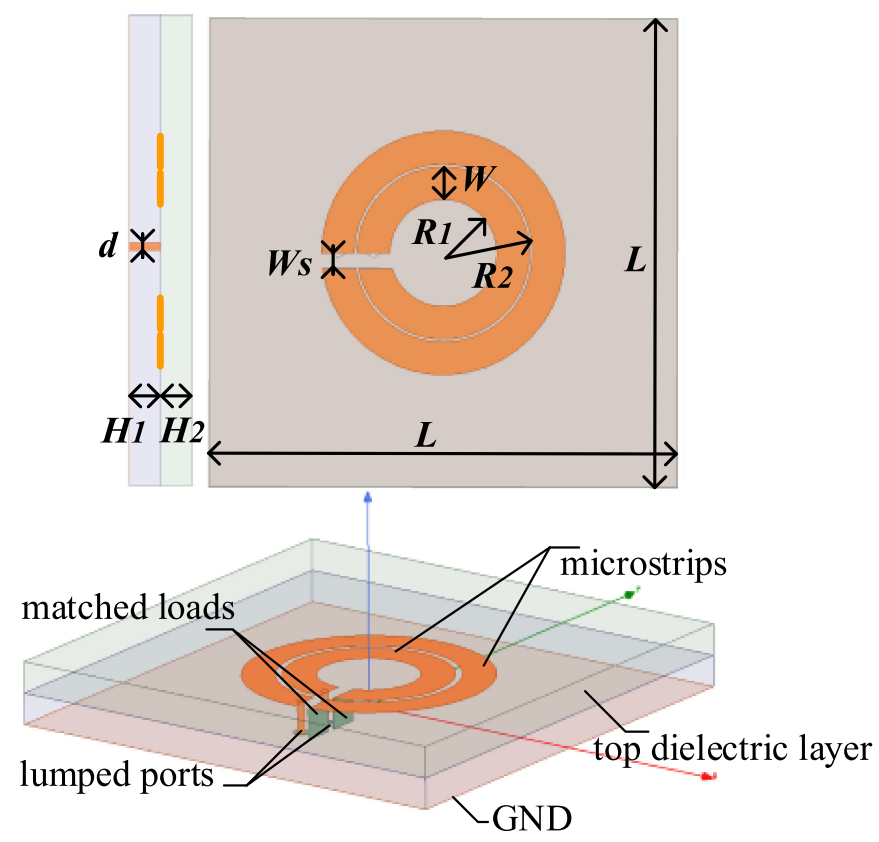

Fig. 1. Simulating configuration of proposed antenna. 
$H_{1}=1 \mathrm{~mm}, H_{2}=0, W=2 \mathrm{~mm}$ and $R_{L}=50 \Omega$, equalling to microstrip's characteristic impedance. On $5 \mathrm{GHz}$, where OAM is generated, its peak gain is $-9.7 \mathrm{~dB}$ for $l=2,-9.3 \mathrm{~dB}$ for $l=3$, and radiation efficiency is 0.02 for $l=2$ and 0.03 for $l=3$, which apparently needs to be improved.

Parameter sweeping shows that values of $W, H_{1}$ and $H_{2}$ have the most significant impact on antenna's radiation efficiency, as shown in Fig. 2. When $W<\lambda_{g} / 4$, larger $W$ leads to higher radiation efficiency. When larger $H_{1}$ is adopted, according to the cavity model of microstrip antenna, its Q factor will decrease and more energy will be radiated to free space, thus its radiation efficiency will be increased. Furthermore, it is also seen that a top dielectric layer has a guiding effect on radiated electromagnetic waves [11], and larger $H_{2}$ brings considerable improvement in radiation efficiency.

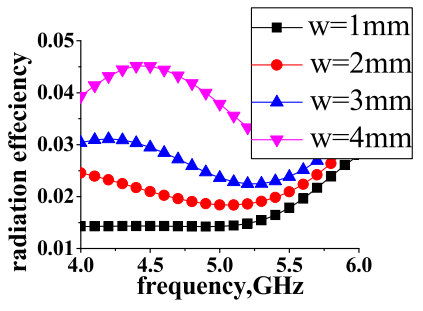

(a)

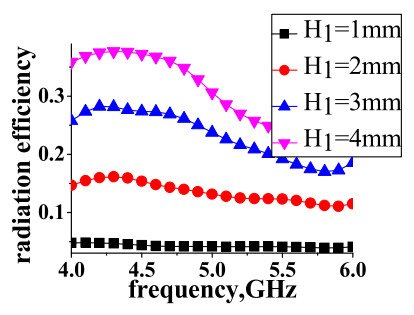

(b)

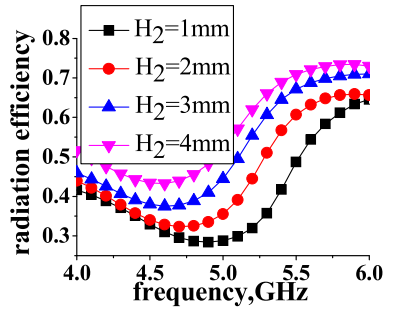

(c)

Fig. 2. Parameter sweeping results of radiation efficiency from 4 to $6 \mathrm{GHz}$. Since the results of $l=3$ are similar, for briefness, only the results of $l=2$ are given.

(a) $W$ is swept while $H_{1}=1 \mathrm{~mm}, H_{2}=0$.

(b) $H_{1}$ is swept while $W=4 \mathrm{~mm}, H_{2}=0$.

(c) $H_{2}$ is swept while $W=4 \mathrm{~mm}, H_{1}=4 \mathrm{~mm}$.

Giving a full consideration to radiation performance and miniaturization, parameters of the proposed antenna are finally optimised as the values in Table I. According to simulation, its peak gain on $5 \mathrm{GHz}$ reaches $2.1 \mathrm{~dB}$ for $l=2$ and $1.9 \mathrm{~dB}$ for $l=3$, greatly improved from the initial values of -9.7 and $-9.3 \mathrm{~dB}$. On a $300 \mathrm{~mm} \times 300 \mathrm{~mm}$ observing plane which is $10 \mathrm{~mm}$ away from antenna's upper surface and parallel to XY plane, phase vortexes of $\boldsymbol{E} \boldsymbol{z}$ for both modes are observed on $5 \mathrm{GHz}$. As shown in the first line of Fig. 3, each vortex has $l$ arms $(l=2$ or 3), and when $\varphi$ varies from $-\pi$ to $\pi$ at any radius, the phase angle will go through the same change $l$ times, which is in agreement with the dependency $e^{j \varphi l}$. Besides, a centre null in radiation patterns is also observed, as shown by arrows in the second line of Fig. 3, conforming to the general case of OAM antennas. However, the nulls are slightly deviated from the centre $\left(\theta=0^{\circ}\right)$ due to the impact of matched loads.

Table I. Optimized parameters

\begin{tabular}{|l|c|c|c|c|c|c|c|c|c|}
\hline Parameters & $L$ & $H_{1}$ & $H_{2}$ & $R_{1}$ & $R_{2}$ & $W$ & $W_{s}$ & $d$ & $R_{L}$ \\
\hline Value $(\mathrm{mm})$ & 60 & 4 & 4 & 6.46 & 11.4 & 4.1 & 2 & 1 & $50 \Omega$ \\
\hline
\end{tabular}



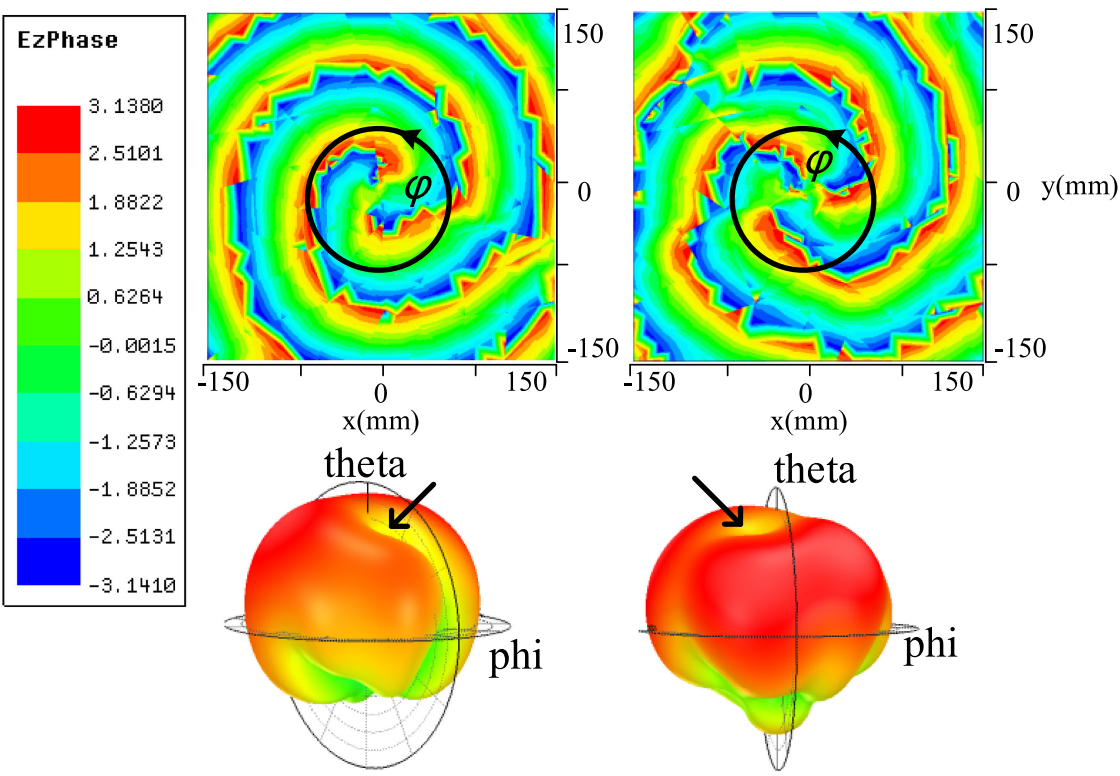

Fig. 3. Simulating results of phase vortexes and $3 \mathrm{D}$ radiation patterns on $5 \mathrm{GHz}$ generated by the antenna with optimised parameters (left column, $l=2$; right column, $l=3$ ). In the phase vortexes at the first line, different colours represent different phase angles from $-\pi$ to $\pi$. Alone the given circle whose center is at the origin, when $\varphi$ varies from $-\pi$ to $\pi$, the colour of phase angles will change from blue to red $l$ times. In the $3 \mathrm{D}$ radiation patterns at the second line, arrows show the centre nulls.

\section{Experimental results}

A prototype antenna is then fabricated and measured according to the optimised parameters in the forward section. As Fig. $4 \mathrm{~b}$ indicates, VSWR of both input ports are acceptable, while the measured results are higher than simulated, especially the input port for inner ring $(l=2)$ on $4-4.5 \mathrm{GHz}$. This is because that near the ground plane, a small part of feeding coaxial cable's inner conductor is exposed to the open air due to fabricating need, resulting in a deviation in cable's characteristic impedance. Fig. $4 c$ shows that the crosstalk of two input ports, $S_{23}$, remains above $13 \mathrm{~dB}$ from 4 to $6 \mathrm{GHz}$.

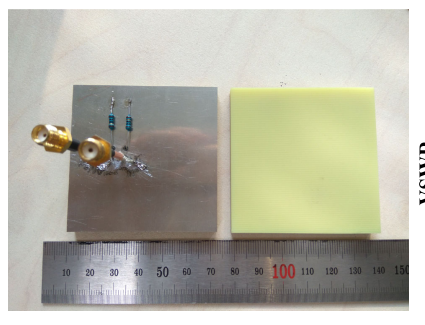

(a)

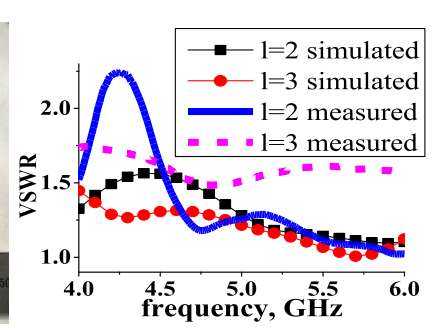

(b)

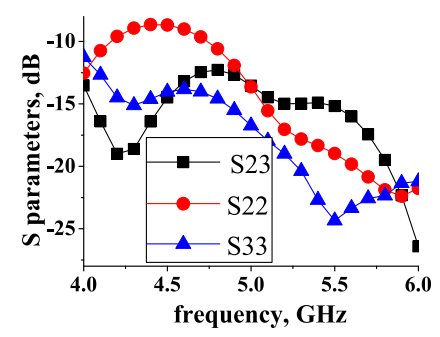

(c)
Fig. 4. Bottom (left) and top (right) view of the prototype antenna (a), measured results of VSWR (b) and S parameters (c). Input port for inner ring is denoted as port 2, and outer as 3 , for they generate OAM with corresponding mode number.

The measured peak gains on $5 \mathrm{GHz}$ are $1.5 \mathrm{~dB}$ for $l=2$ and $1.8 \mathrm{~dB}$ for $l=3$, lower than simulated values, but still show an improvement of more than $10 \mathrm{~dB}$ 
compared with simulating results of the initial model without any optimization. As shown in the measured radiation patterns on E plane in Fig. 5, agreeing with the simulating results, centre nulls are observed in both modes, while they are slightly deviated from $\theta=0^{\circ}$ due to matched loads, especially when $l=3$.

Finally, the phase of $\boldsymbol{E} \boldsymbol{z}$ generated by the prototype antenna is measured on an observation plane of $1 \mathrm{~m} \times 1 \mathrm{~m}, 1 \mathrm{~m}$ away from antenna's top surface. Phase vortexes similar as simulating results are successfully generated, from 4.65 to $5.25 \mathrm{GHz}$ for $l=2$, and 4.5 to $5.2 \mathrm{GHz}$ for $l=3$, when the inner and outer rings are respectively fed. The results are plotted in five scales, which are represented by different colours. Due to the noise and the insufficient scale number, which is restricted by our limited measurement conditions, some discontinuities occur in the measured phase vortexes. However, the $l$-arm vortexes, and the property that when $\varphi$ varies from $-\pi$ to $\pi$ at any radius, the phase angle will go through the same change $l$ times, are still distinguishable.

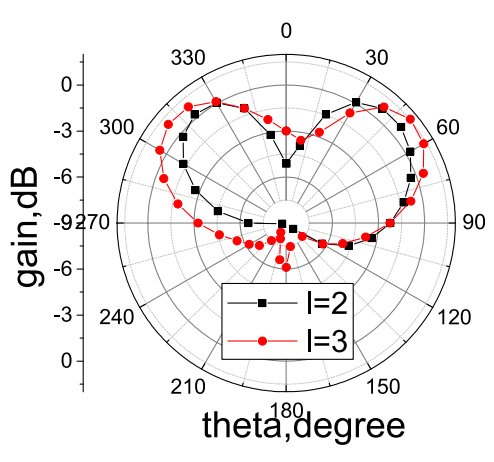

theta,degree

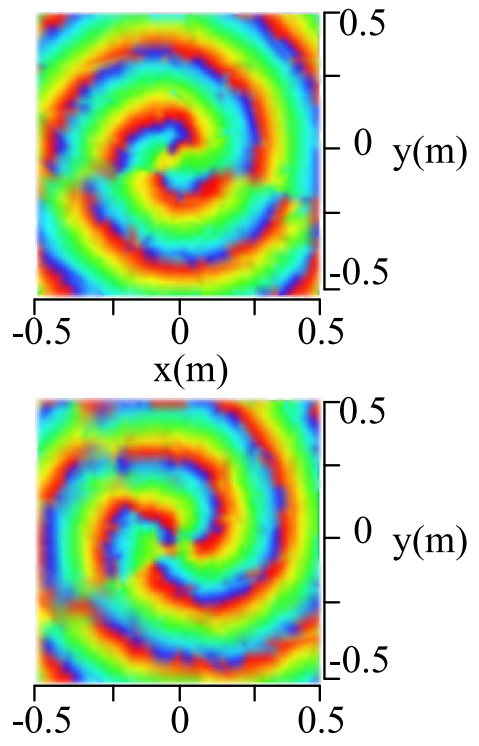

Fig. 5. Measured results of $\mathrm{E}$ plane radiation patterns and phase vortexes on $5 \mathrm{GHz}$.

\section{Conclusion}

A double-OAM-mode microstrip antenna has been presented. Compared with existing OAM antennas, it is especially convenient to be integrated in RF circuit and electronic devices for OAM wireless communication. Besides, it can also multiplex more OAM modes by adopting more microstrip rings. Based on circular microstrip line, its parameters are optimized and a top dielectric layer is adopted to improve radiation efficiency and gain.

\section{Acknowledgments}

The work was supported by the National Natural Science Foundation of China (grant no. 61771490 and 61302051), and by Basic Foundation of Engineering University of PAP (grant no. WJY201606). 